\title{
Dulaglutide as Add-on Therapy to SGLT-2 Inhibitors in Patients With Inadequately Controlled Type 2 Diabetes (AWARD-10): A 24-Week, Randomised, Double-Blind, Placebo-Controlled Trial
}

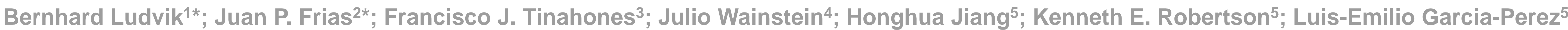
D. Bradley Woodward ${ }^{5}$; Zvonko Milicevic ${ }^{6}$; Roy Rasalam?

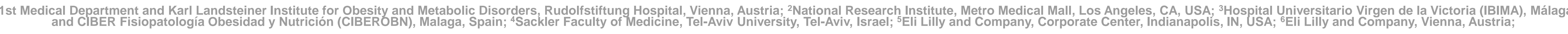

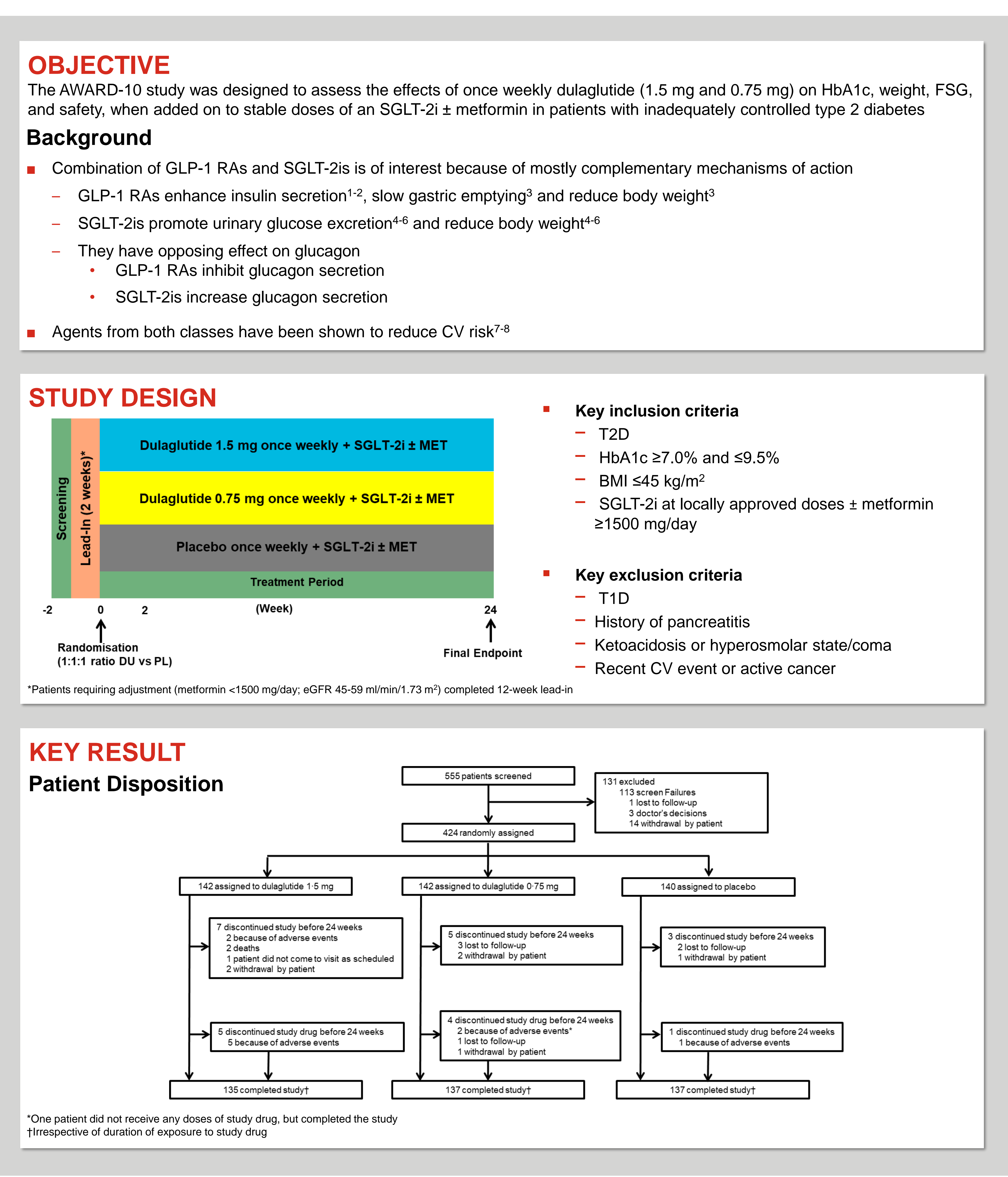

Methods

Primary objective

Primary objective was to demonstrtate superiority of addition of dulaglutidid vis
SGLT-2is tor change trom baseline in HbAlc after 24 weeks of treatment

Other objectives at 24 weeks

- Secondary efficacy objectives

Percentage of patients achieving $\mathrm{HbA1c}$ target of $<7.0 \%$ and
$56.5 \%$

Change in body weight

Change in FSG

Change in SMPG profile

Change in fasting glucagon

- Secondary safety objectives

Statistical analysis

- Graphical testing app

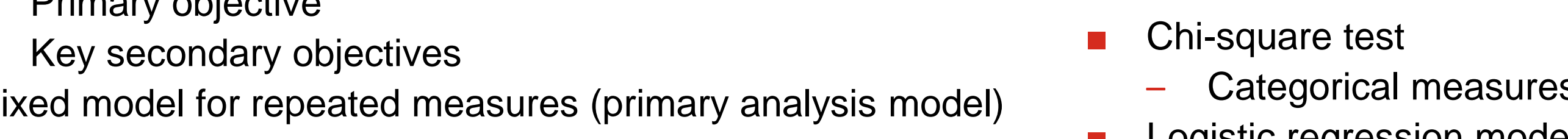
Body weight
Vital sign data
A alysis of covariance model
FSG
Glucagon
Pegistic regression model
Generalized linear model with negative binomial distribution Hypoglycaemia rate

Baseline characteristics

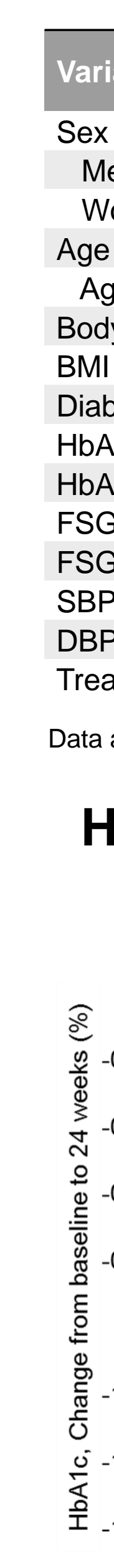

Adverse events, vitals, ECGs, hypoglycemia

Adjudicated pancreatitis, CV events

- Percentage of patients achieving HbA1c target $<7.0 \%$ with no weight gain and no documented symptomatic hypoglycaemia Percentage of patients achieving HbA1c target $<7.0 \%$ with body weight loss
hypoglycaemia
Percentage of patients achieving HbA1c targets at 24 weeks

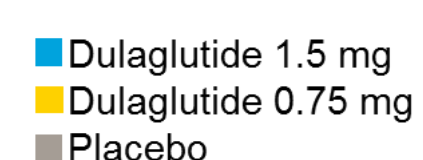

Weight, change to 24 weeks

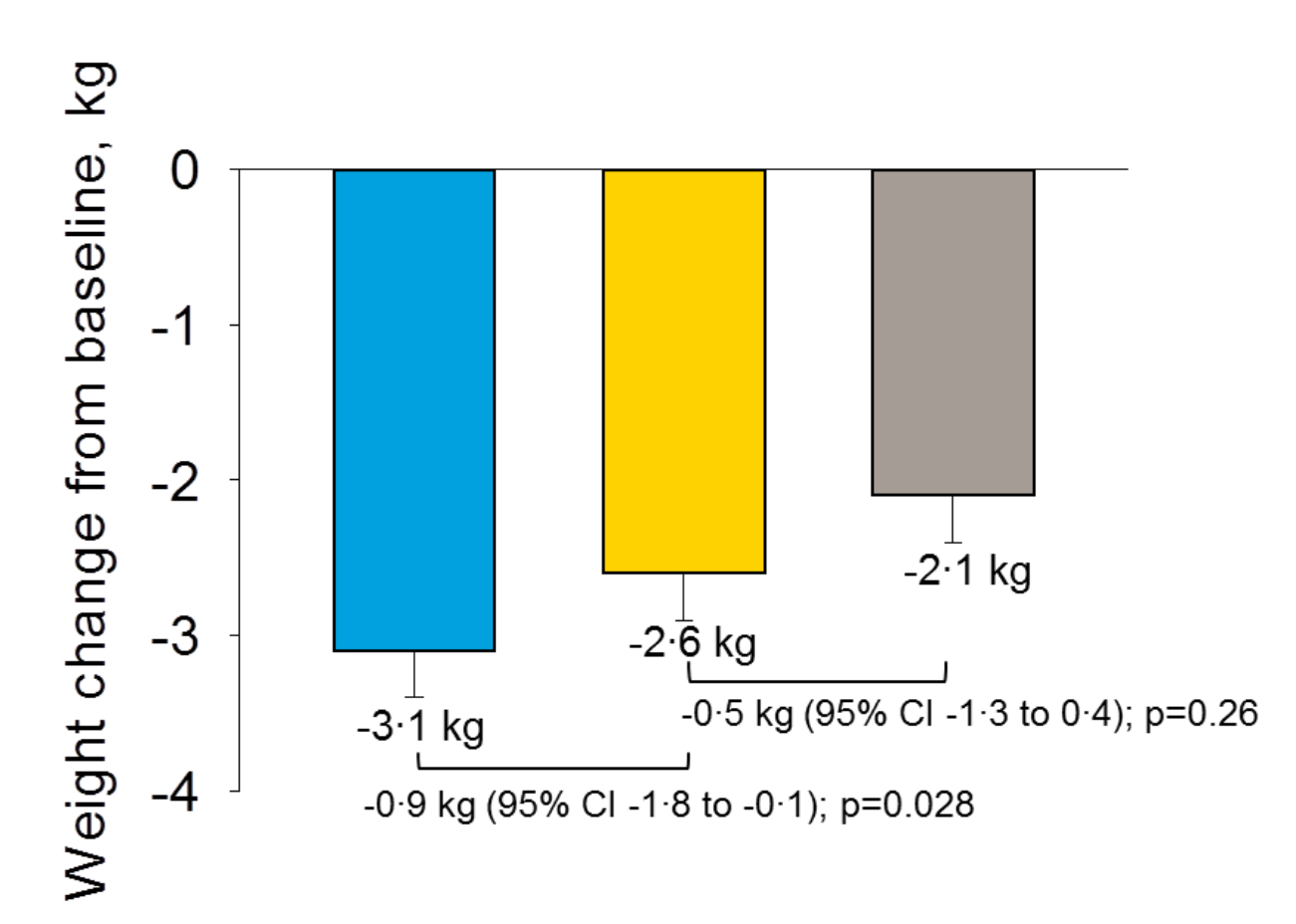

Composite endpoints at 24 weeks

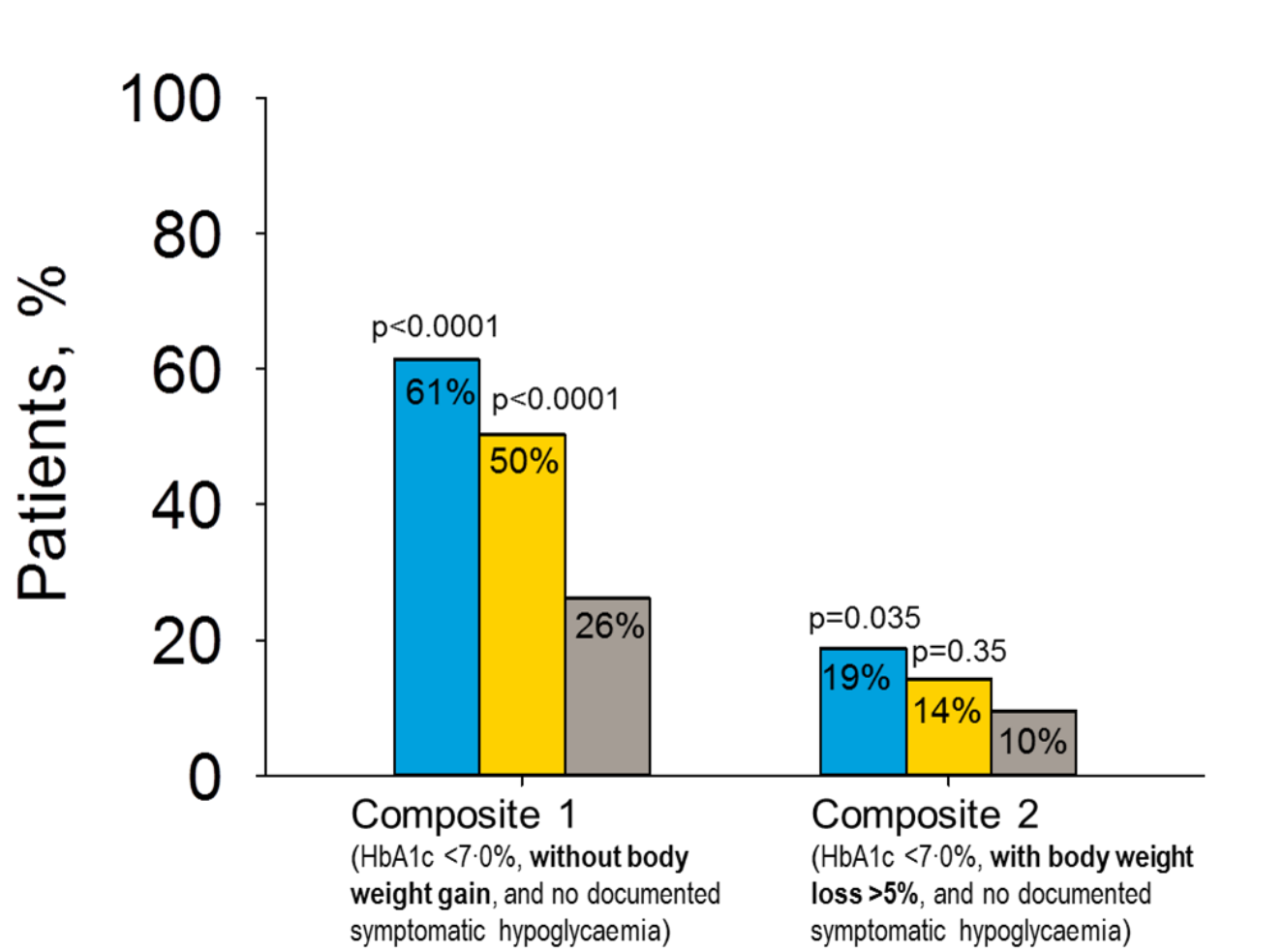

Adverse events through 24 weeks

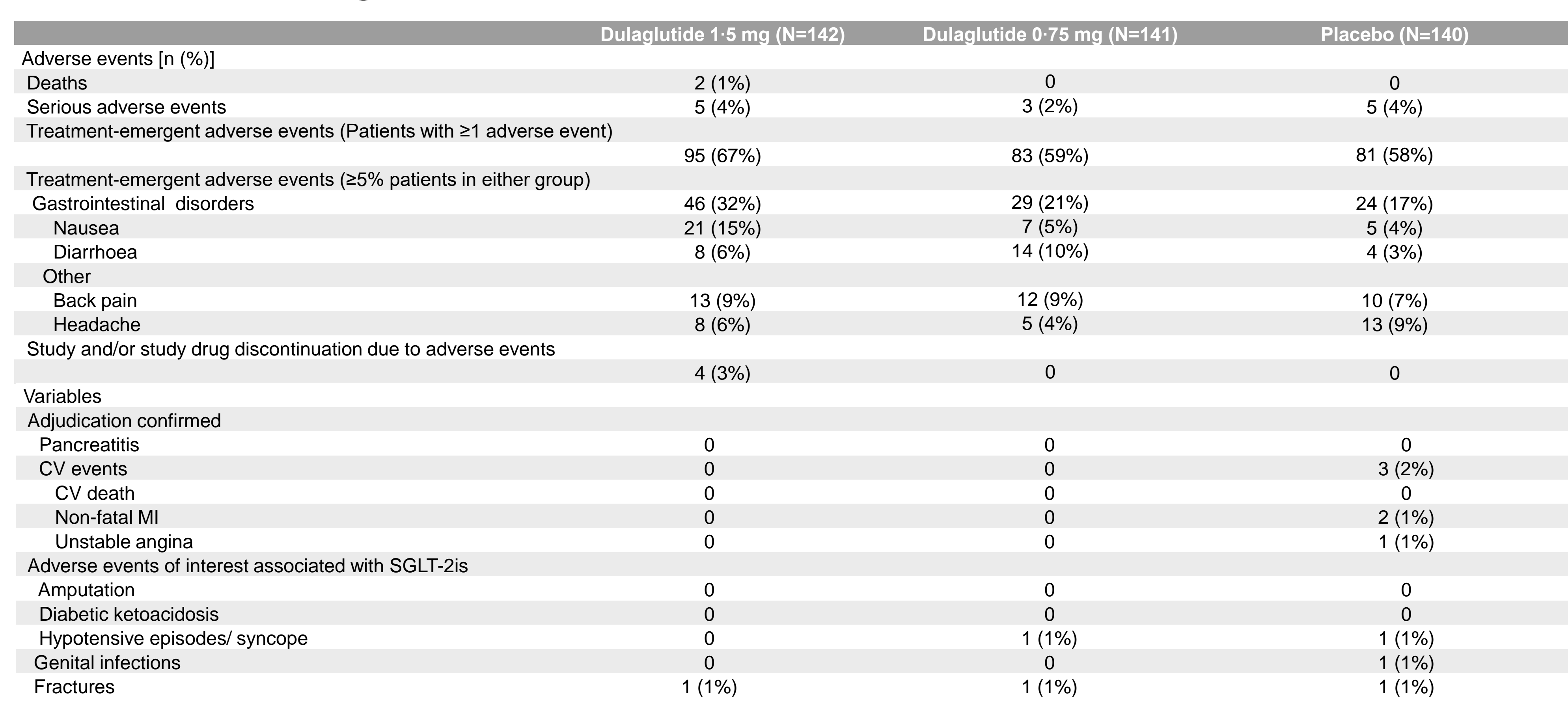

Hypoglycaemia through 24 weeks

\begin{tabular}{|c|c|c|c|}
\hline 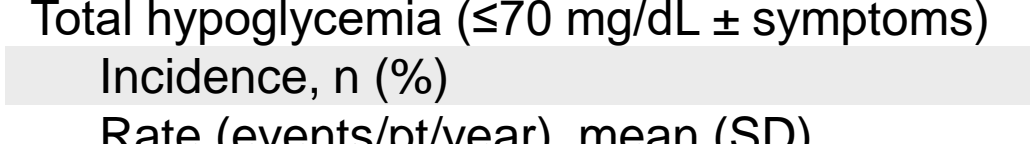 & & & \\
\hline $\begin{array}{l}\text { Inciaence, nolvo)ear), mean (SD) } \\
\text { Rate (events(ty) } \\
\text { 30-day rate, mean (SD) }\end{array}$ & $\begin{array}{l}0.31(2.22) \\
0.31 \\
0.03018\end{array}$ & $\begin{array}{l}0.26 .0 .67) \\
002(1.674)\end{array}$ & $\begin{array}{l}0.21(1.61) \\
0.02(0.13)\end{array}$ \\
\hline $\begin{array}{l}\text { Documented symptomatic ( } \leq 70 \mathrm{mg} / \mathrm{LL}) \\
\text { Incidence ( }(\%)\end{array}$ & & & \\
\hline 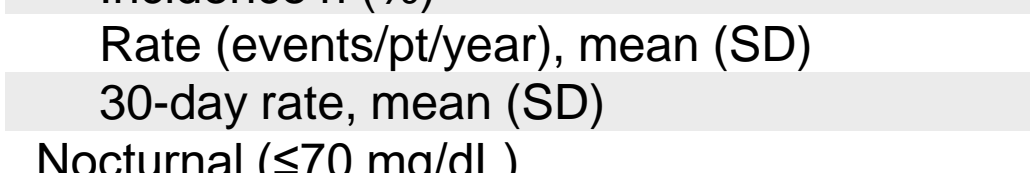 & $\begin{array}{l}0.16(1.71) \\
0.01(0.14)\end{array}$ & $\begin{array}{l}0.16(1.25) \\
0.01(0.10)\end{array}$ & $\begin{array}{l}0.12(1.09) \\
0.01(1.09)\end{array}$ \\
\hline 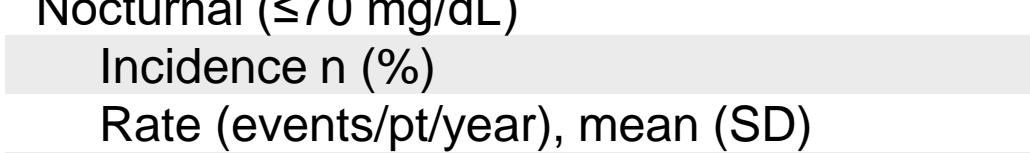 & $\begin{array}{c}1(0.7) \\
0.03(0.35)\end{array}$ & $\begin{array}{c}2(1.4) \\
0.11(1.00)\end{array}$ & $\begin{array}{l}0(0.0) \\
0.00(0.0)\end{array}$ \\
\hline $\begin{array}{l}\text { 30-day rate, mean (SD) } \\
\text { Severe hypoglycemia, } \mathrm{n}(\%)\end{array}$ & $\begin{array}{l}0.002(0.03) \\
0(0.0)\end{array}$ & $\begin{array}{l}0.01(0.08) \\
1(0.7)\end{array}$ & $\begin{array}{l}0.00(0.00) \\
0(0.0)\end{array}$ \\
\hline
\end{tabular}

Summary of baseline SGLT-2i dose

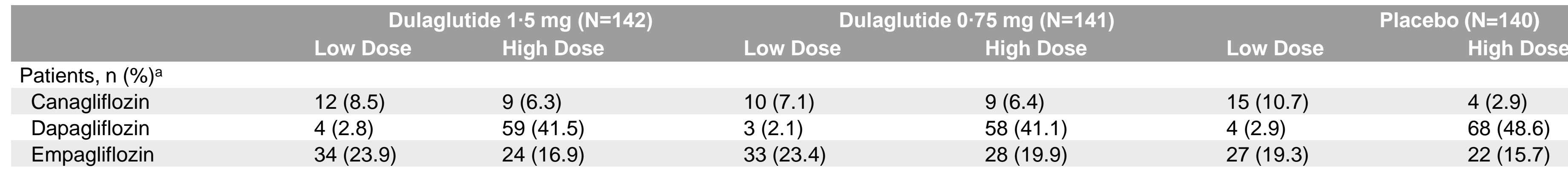

Summary

- The addition of dulaglutide to ongoing SGLT-2i treatment \pm metformin resulted in statistically significant and clinically relevant reduction

- Dulaglutide $1.5 \mathrm{mg}$ dose resulted in significantly greater reduction in body weight versus placebo

- Treatment with dulaglutide was associated with higher incidence of gastrointestinal adverse events

- Dulaglutide $1.5 \mathrm{mg}$ significantly decreased SBP from baseline versus placebo

Limitations

- Short duration (24 weeks)

Patients were inadequately controlled on SGLT-2i \pm metformin $\geq 1500 \mathrm{mg} /$ day as tolerated, thus the results cannot be generalised to patients who do not meet these criteria

- Most patients had been taking a SGLT-2i for less than six months prior to enrolling in the study, which may partially explain a statistically

- This study did not include a placebo-only group (all treatment groups received SGLT-2i \pm metformin) to inform the contributions of

A substantial change from baseline for HbA1c in the placebo group was observed

\section{CONCLUSIONS}

- In AWARD-10 once weekly dulaglit

- These results showed that in patients

effective and safe treatment option

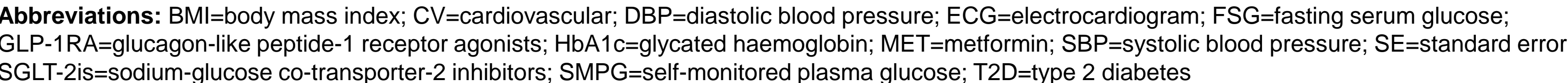

1. Holst tetal. FEBS Letters 1987:211 (2): 196-74.

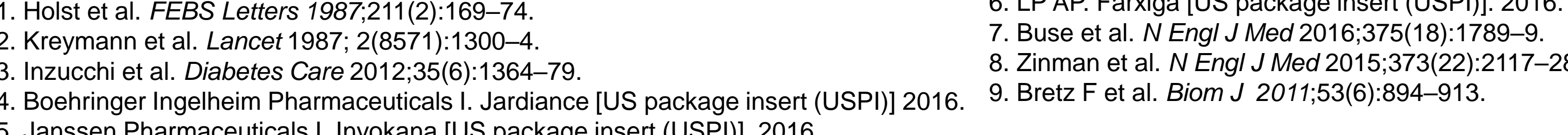

